\title{
A Case of Amniotic Band Sequence Complicated by Severe Skeletal Malocclusion
}

\author{
Sayaka Yoshiba ${ }^{*}$, Tetsutaro Yamaguchi², Sumimasa Ohtsuka ${ }^{3}$, Tatsuo Shirota ${ }^{1}$ \\ ${ }^{1}$ Department of Oral and Maxillofacial Surgery, School of Dentistry, Showa University, Tokyo, Japan \\ ${ }^{2}$ Department of Orthodontics, School of Dentistry, Showa University, Tokyo, Japan \\ ${ }^{3}$ Ohtsuka Orthodontic Clinic, Tokyo, Japan \\ Email: *yoshiba@dent.showa-u.ac.jp
}

Received 23 March 2015; accepted 12 April 2015; published 17 April 2015

Copyright (C) 2015 by authors and Scientific Research Publishing Inc.

This work is licensed under the Creative Commons Attribution International License (CC BY).

http://creativecommons.org/licenses/by/4.0/

c) (i) Open Access

\section{Abstract}

This clinical report describes the management of a patient who was diagnosed with amniotic band sequence (ABS) complicated with severe skeletal malocclusion, associated with severe muscle weakness. The patient was a 23-year-old male who underwent treatment in our hospital to improve chewing, phonation, and swallowing difficulties caused by severe abnormality of the maxillofacial skeleton. We evaluated chewing, swallowing and language functions preoperatively, and started muscle function and oral rehabilitation therapy. We performed 2-jaw osteotomy after preoperative orthodontic treatment. The patient continued with postoperative orthodontic treatment and oral rehabilitation. Postoperatively, the patient showed improved maxillofacial morphology, but was unable to attain sufficient improvement of masticatory and language functions due to atrophy and poor development of the masticatory muscles. These results suggest that obtaining sufficient therapeutic effect on oral functions is not possible if the masticatory muscles are not sufficiently strong due to atrophy or hypoplasia.

\section{Keywords}

Amniotic Band Sequence, Osteotomy, Skeletal Malocclusion

\section{Introduction}

Amniotic band sequence (ABS) is caused by rupture of the amniotic membrane in the early embryonic stage,

\footnotetext{
"Corresponding author.
}

How to cite this paper: Yoshiba, S., Yamaguchi, T., Ohtsuka, S. and Shirota, T. (2015) A Case of Amniotic Band Sequence Complicated by Severe Skeletal Malocclusion. Case Reports in Clinical Medicine, 4, 124-130. 
with insufficient amniotic fluid leading to the formation of cord-like structures that can create a variety of malformations in the fetus [1] [2]. This syndrome is sporadic, reportedly occurring in approximately 1 in 12,000 15,000 live births [3]. The clinical manifestations of ABS vary from death secondary to disruption of the umbilical cord or anencephaly, to amputations of extremities, facial cleft or lymphedema [4]-[7].

We encountered a case of ABS in which the patient exhibited severe skeletal dysfunction associated with muscle weakness. To the best of our knowledge, this represents the first case of ABS described in the literature characterized by severe skeletal malocclusion and managed by 2-jaw osteotomy with pre- and postoperative functional evaluations.

\section{Case Report}

A 23-year-old man was referred to our department complaining of severe masticatory, language and swallowing dysfunctions due to abnormalities of the maxillofacial skeleton. His medical history included ABS and congenital nasopharyngeal regurgitation. His family history was unremarkable. He had been diagnosed with ABS at 1 year old and underwent a pharyngeal flap operation in a hospital of plastic surgery. He then visited our hospital in April 1992 for the treatment of feeding and phonate difficulties, and started orthodontic treatment in July 2009.

Preoperative extraoral examination showed reversed occlusion and deformation of the facial appearance (Figure 1, Figure 2). In addition, constriction bands and deformation of the limbs were identified (Figure 3). Preoperative intraoral findings exhibited the angle class two malocclusion and severe skeletal open bite (Figure 2). The patient could not speak his lines smoothly and maintain a closed position of the jaws due to weakness of the masticatory muscles. Preoperative cephalometric analysis showed poor growth of the maxilla and false mandibular prognathism (Table 1). MRI showed atrophy of the masticatory muscles (Figure 4). Severe muscle weakness induced poor development of the mandibular ramus, resulting in severe open bite (Figure 5). We therefore attempted to achieve functional recovery using muscle function therapy pre- and postoperatively. We performed Lefort type I osteotomy and mandibular sagittal splitting osteotomy under general anesthesia in May 2010. We placed two bone screws on the maxilla and mandible and performed inter-maxillary fixation for 1 week from postoperative day 2. Since preservation of the teeth was a key consideration, ideal overbite was not apparent on postoperative intraoral examination. With the release of intermaxillary fixation, occlusion was not stable. We performed objective therapeutic evaluations before and after surgery using electromyography (EMG) and tests of masticatory and language functions.

EMG was carried out for the left and right masseter muscles and anterior and posterior areas of temporalis muscle at maximum clenching in the intercuspal position, using an MP100 system (BIOPACK System, CA, USA) at a frequency of $1 \mathrm{kHz}$. Dedicated software (AcqKowledge ${ }^{\circledR}$ version 3.2.7.; BIOPACK System) was used for analysis. EMG findings showed no significant changes in voluntary muscle activity of jaw-closing between before and after surgery (Figure 6). Masticatory function was assessed using an evaluation sheet for chewing function [8]. Preoperative functional score improved from $10 \%$ to $45 \%$ after surgery (Table 2).

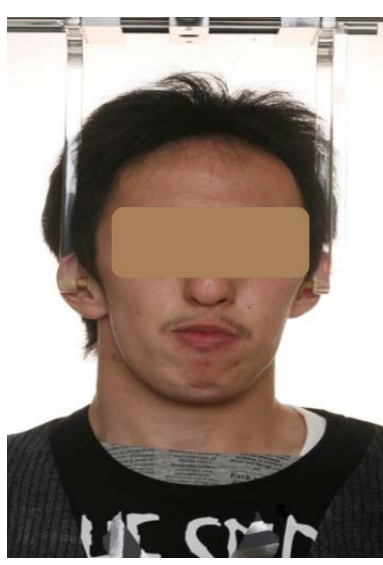

(a)

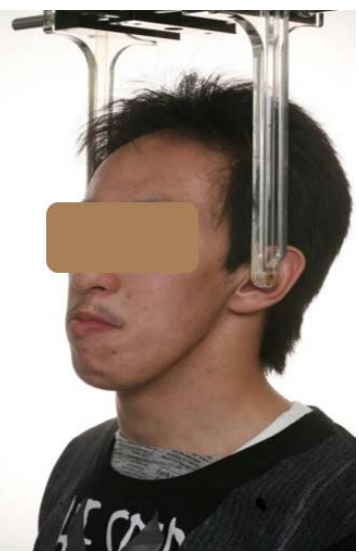

(b)

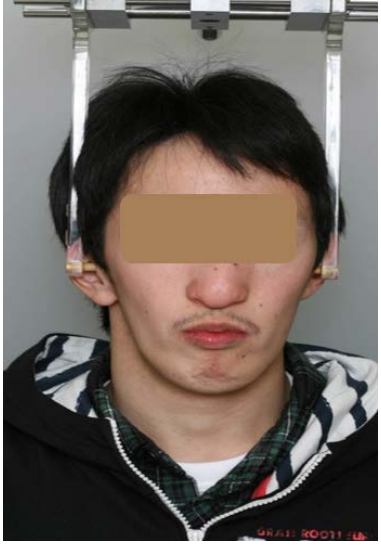

(c)

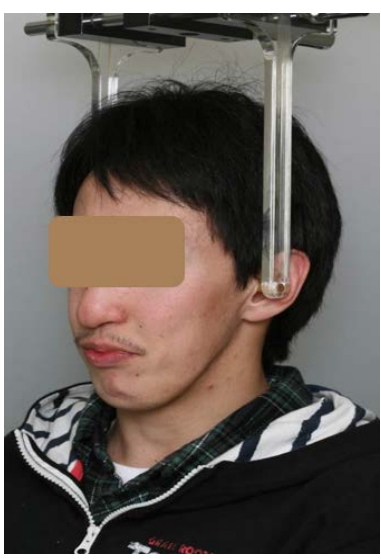

(d)

Figure 1. (a), (b) Preoperative extra-oral examination shows a high degree of skeletal malocclusion; (c), (d) Postoperative extra-oral examination shows improved craniofacial morphology. 


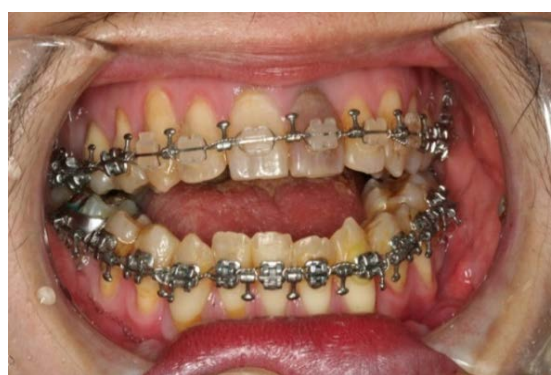

(a)

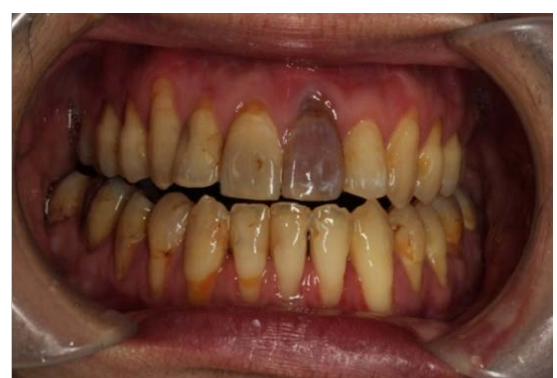

(b)

Figure 2. (a) Preoperative intra-oral examination shows severe reversed occlusion; (b) Postoperative intra-oral examination does not show acquisition of ideal overbite, as this goal was not sought in consideration of preservation of the teeth.

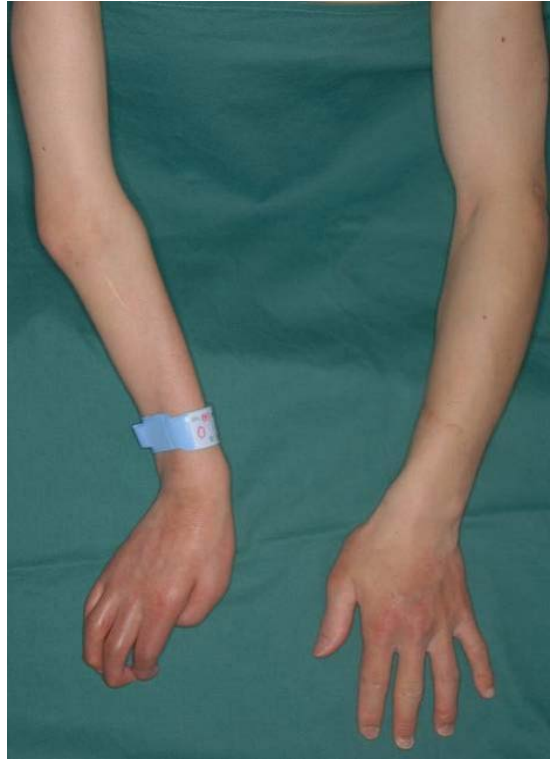

(a)

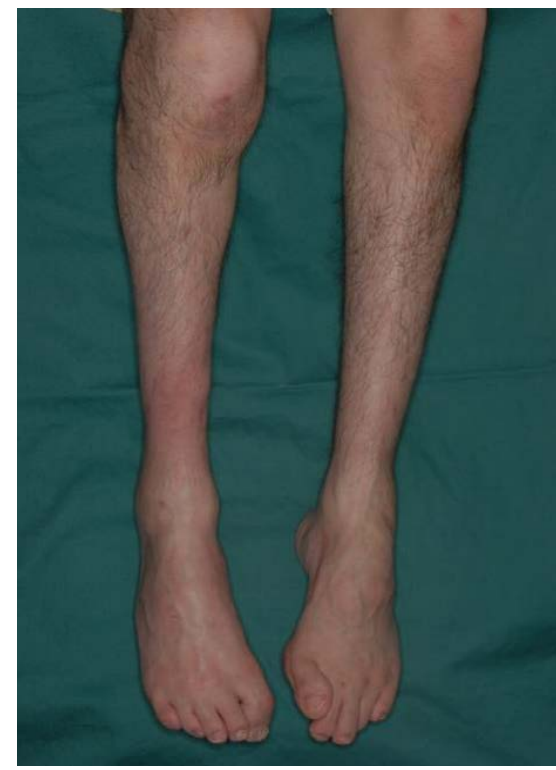

(b)

Figure 3. Constriction band and deformation of the extremities of the patient.

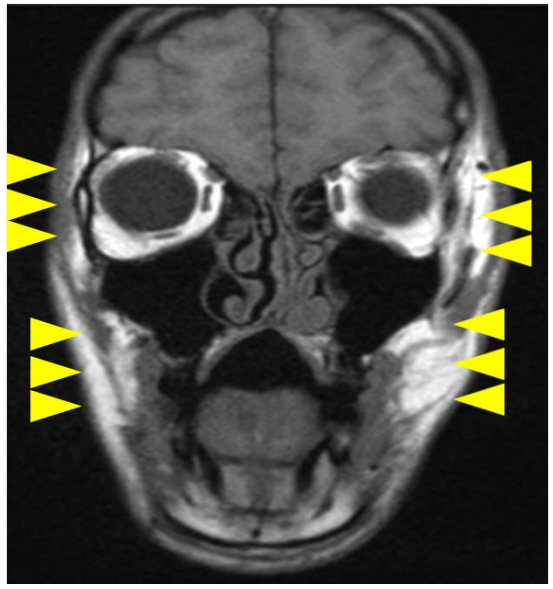

(a)

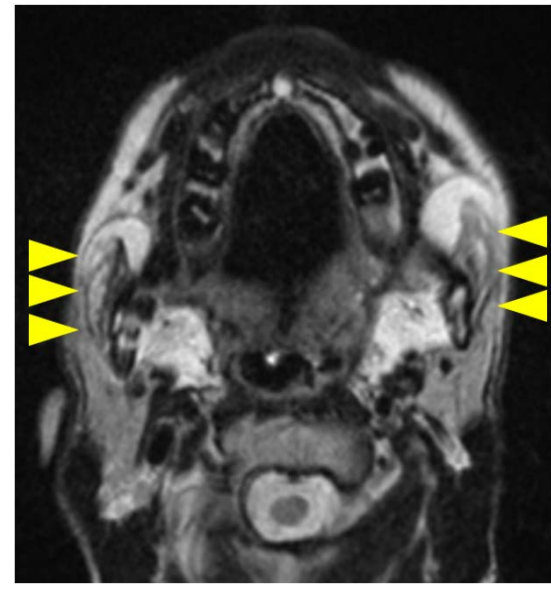

(b)

Figure 4. Postoperative MRI shows atrophy and poor development of bilateral masticatory muscles. 


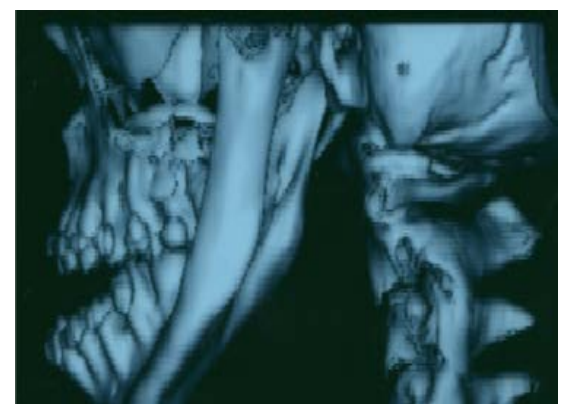

(a)

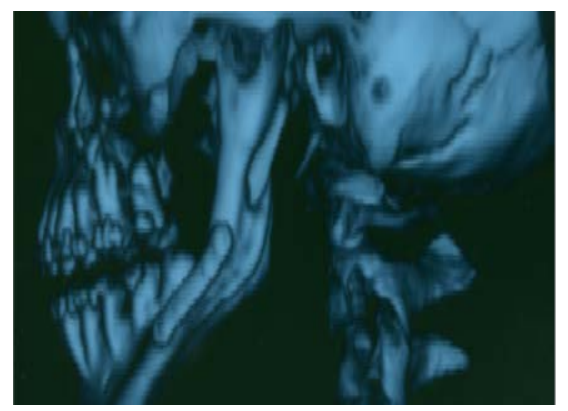

(b)

Figure 5. (a) Preoperative CT shows thinning of the maxillary jaw and very thin form of the mandibular ramus; (b) Postoperative CT shows improved craniofacial morphology.

Table 1. Pre- and postoperative cephalometric analyses.

\begin{tabular}{|c|c|c|c|}
\hline & Mean \pm S.D. & Pre-operation & Post-operation \\
\hline \multicolumn{4}{|l|}{ Angular analysis } \\
\hline SNA & $81.12 \pm 3.09$ & 78.24 & 86.08 \\
\hline SNB & $76.81 \pm 3.14$ & 76.76 & 79.84 \\
\hline ANB & $3.28 \pm 2.66$ & 1.49 & 6.25 \\
\hline $\mathrm{FH}$ to $\mathrm{SN}$ & $5.98 \pm 3.35$ & 4.24 & 4.98 \\
\hline Facial Angle & $85.07 \pm 5.76$ & 80.66 & 85.32 \\
\hline Convexity & $5.6 \pm 4.33$ & 3.9 & 11.87 \\
\hline A-B Plane & $-5.1 \pm 3.28$ & -1.58 & -9.27 \\
\hline Mandibular plane & $26.25 \pm 6.34$ & 60.78 & 47.21 \\
\hline Gonial Angle & $116.3 \pm 8.8$ & 159.79 & 153.49 \\
\hline Occlusal plane & $9.52 \pm 4.01$ & 22.95 & 18.14 \\
\hline \multicolumn{4}{|l|}{ Linear analysis } \\
\hline $\mathrm{N}-\mathrm{S}$ & $71.85 \pm 2.58$ & 68.35 & 69.18 \\
\hline N-ME & $136.11 \pm 5.68$ & 145.93 & 135.94 \\
\hline GN-CD & $128.52 \pm 4.39$ & 122.13 & 121.06 \\
\hline POG'-GO & $82.05 \pm 3.76$ & 63.9 & 76.23 \\
\hline CD-GO & $69.62 \pm 4.89$ & 53.99 & 48.13 \\
\hline IS-IS' & $32.6 \pm 2.82$ & 31.5 & 30.63 \\
\hline MO-MS' & $26.96 \pm 1.98$ & 28.25 & 26.05 \\
\hline II-II' & $48.71 \pm 1.58$ & 44.59 & 44.86 \\
\hline MO-MI' & $37.04 \pm 2.15$ & 26.27 & 32.61 \\
\hline
\end{tabular}




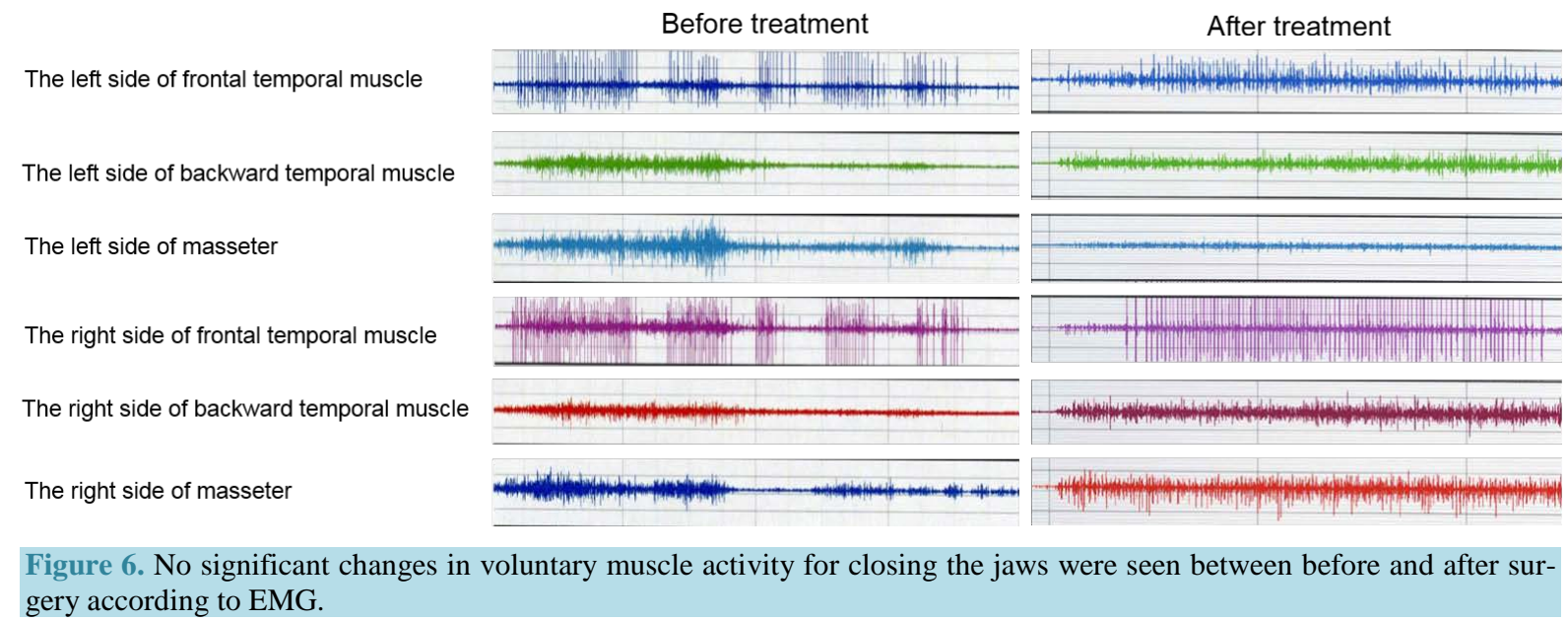

Table 2. Assessment of chewing function in complete denture wearers, based on the food intake questionnaire method. $\circ$ : Easy to chew; $\Delta$ : difficult to chew; $\times$ : impossible to chew. Chewing factor score $=($ number of $\circ / 20) \times 100$.

\begin{tabular}{|c|c|c|c|}
\hline Class & Food & Before treatment & After treatment \\
\hline \multirow{4}{*}{1} & Whole apple & $\times$ & $\times$ \\
\hline & Chewing gum & $x$ & $x$ \\
\hline & Dried shell ligament & $x$ & $x$ \\
\hline & Dried cuttlefish & $x$ & $x$ \\
\hline \multirow{4}{*}{2} & Fresh ear shell & $x$ & $\times$ \\
\hline & Hard pickled radish & $x$ & $\triangle$ \\
\hline & Hard cracker & $\triangle$ & $\triangle$ \\
\hline & Hard biscuit & $\triangle$ & $\triangle$ \\
\hline \multirow{4}{*}{3} & Pickled radish & $\triangle$ & $\triangle$ \\
\hline & Peanuts & $\triangle$ & $\triangle$ \\
\hline & Beef steak & $\triangle$ & ० \\
\hline & Rice-cake cubes & $\triangle$ & ० \\
\hline \multirow{4}{*}{4} & Burdock & $\triangle$ & $\triangle$ \\
\hline & Potato chips & $\triangle$ & ० \\
\hline & Boiled fish paste (kamaboko) & $\triangle$ & ० \\
\hline & Artificially grown soybean & ० & ० \\
\hline \multirow{4}{*}{5} & Boiled carrot & $\triangle$ & ० \\
\hline & Boiled potato & $\triangle$ & ० \\
\hline & Boiled eggplant & $\triangle$ & ० \\
\hline & Bean curd (Tofu) & 0 & 0 \\
\hline
\end{tabular}


Speech function was assessed using the standard Japanese language test and conversation intelligibility test. The standard Japanese language test was carried out in Japanese. Each patient was instructed to pronounce 100 syllables in front of the recorder. The five listeners transcribed the sounds produced by the patient as the Japanese syllables they believed they had heard. The overall intelligibility score was expressed as the mean percentage of correct responses for all five listeners [9]. Scores were also assessed on the basis of articulatory points, divided into six groups: bilabial; dental-alveolar; palatal-alveolar; palatal; velar; and glottal sounds.

In the Japanese intelligibility speech test for 100 syllables, the overall percentage of correct answers improved from $22.4 \%$ preoperatively to $26.4 \%$ postoperatively. In terms by articulation, dental-alveolar, alveolar and velar sounds showed the greatest improvements (Table 3).

In the conversation intelligibility test, healthy five individuals listened to the conversation of the patient, then provided evaluations using a 5-point rating score, with higher score indicating less clear contents of the conversation. Conversation intelligibility test results improved from 3.6 to 2.8. Craniofacial morphology improved with 2-jaw osteotomy, but the patient did not show sufficient functional recovery.

\section{Discussion}

Even though the mechanisms causing ABS remain unclear, the most widely accepted theory is that rupture of the amniotic sac early in pregnancy results in the formation of fibrous bands of mesoderm originating from the chorionic side of the amnion. These bands then disrupt growth when they become entangled with the fetus. ABS is a sporadic condition that occurs in approximately one in 12,000 - 15,000 births [1]-[3].

Although the precise mechanism for development of oro-facial anomalies associated with amniotic band syndrome is not fully understood, two main theories of the pathogenesis of ammonic band syndrome namely the extrinsic model and intrinsic model [6] [7]. The extrinsic model holds that an early rupture of the ammonic sac causes the formation of amniochorionic mesodermal bands leading to the development of amniotic construction band. Early rupture may decrease amniotic fluid, causing the compressive consequences of early constraint, such as scoliosis and clubfoot. Additionally, and more severely, a vascular disruption can result from the early rupture, leading to facial clefts and limb reduction with body-wall defect. The intrinsic model holds that an intrinsic, germline developmental abnormality is responsible for the development of amniotic construction band. This theory is often used to explain major craniofacial abnormalities, body-wall defects, and internal organ abnormalities. While other theories may be more convincing in their ability to explain extremity abnormalities, the intrinsic model remains a popular means of explaining the central abnormalities. Morphological abnormalities of this disease abound, and depend on the time of rupture of the amniotic membrane. Therefore, each of the theories better explains a subset of ABS cases, reflecting that ABS is etiologically heterogeneous.

In our case, the patient exhibited a false mandibular prognathism caused by poor growth of the maxilla. The

Table 3. Results of pre- and postoperative Japanese syllable speech intelligibility testing. Overall percentage of correct answers improved from $22.4 \%$ to $26.4 \%$, with scores for articulation, sibilant, alveolar and velar sounds showing the most improvement.

\begin{tabular}{ccc}
\hline Variable & Before treatment (\%) & After treatment (\%) \\
\hline Overall correct answer & 22.4 & 26.4 \\
Bilabial & 23.8 & 21.5 \\
Dental-alveolar & 15.6 & 31.9 \\
Palato-alveolar & 10 & 8.8 \\
Palatal & 37.1 & 31.4 \\
Velar & 3.8 & 15 \\
Glottal & 100 & 80 \\
Vowels & 84 & 76 \\
\hline
\end{tabular}


patient showed dysfunction of the masticatory muscles on the initial visit to our hospital and we conducted functional recovery therapy for muscle function both before and after surgery. We evaluated the therapeutic effects objectively pre- and postoperatively using EMG and tests of masticatory and language functions. EMG results showed no significant change between before and after surgery. Preoperative functional score was $10 \%$, improving to $45 \%$ after surgery (Table 2). With the Japanese 100-syllable intelligibility speech test, the overall percentage of correct answers improved from $22.4 \%$ to $26.4 \%$. In terms of articulation, sibilant, alveolar and velar sounds were the most improved (Table 3). Results of the conversation intelligibility test improved from 3.6 preoperatively to 2.8 postoperatively. Craniofacial morphology improved with the 2-jaw osteotomy, but the patient did not achieve adequate functional recovery. This was attributed to poor growth and atrophy of the masticatory muscles as seen on MRI. Sufficient activation of the muscles of mastication in rehabilitation may not be achievable beyond a certain threshold of atrophy. Because the maxillofacial skeleton was improved with orthgnathic surgery, postoperative function of the surrounding masticatory muscles was not improved.

\section{Conclusion}

This represents the first report of osteotomy using pre- and postoperative muscle functional therapy in a patient with ABS. The craniofacial morphology was improved with orthognathic surgery, but careful observation of the stability of postoperative occlusion will be needed due to the inadequate muscle activity of the masticatory muscles.

\section{Acknowledgements}

No grants were received in relation to this paper.

\section{Competing Interests}

The authors declare that they have no competing interests.

\section{References}

[1] Taub, P.J., Lin, H. and Silver, L. (2007) Mandibular Distraction for Amniotic Band Syndrome in the Neonate. Annals of Plastic Surgery, 59, 334-337. http://dx.doi.org/10.1097/01.sap.0000252771.42869.b2

[2] Kim, J., Hollier, L. and Taylor, T. (2004) Amniotic Band Sequence: The Use of Bone Grafting and Distraction Osteogenesis. Journal of Craniofacial Surgery, 15, 347-351. http://dx.doi.org/10.1097/00001665-200403000-00035

[3] Cignini, P., Giorlandino, C., Padula, F., Dugo, N., Cafà, E.V. and Spata, A. (2012) Epidemiology and Risk Factors of Amniotic Band Syndrome, or ADAM Sequence. Journal of Prenatal Medicine, 6, 59-63.

[4] Doi, Y., Kawamata, H., Asano, K. and Imai, Y. (2011) A Case of Amniotic Band Syndrome with Cleft Lip and Palate. Journal of Maxillofacial and Oral Surgery, 10, 354-356. http://dx.doi.org/10.1007/s12663-011-0174-4

[5] Muraskas, J.K., McDonnell, J.F., Chudik, R.J., Salyer, K.E. and Glynn, L. (2003) Amniotic Band Syndrome with Significant Orofacial Clefts and Disruptions and Distortions of Craniofacial Structures. Journal of Pediatric Surgery, 38, 635-638. http://dx.doi.org/10.1053/jpsu.2003.50141

[6] Kahramaner, Z., Cosar, H., Turkoglu, E., Erdemir, A., Kanik, A., Sutcuoglu, S. and Ozer, E.A. (2012) Amniotic Band Sequence: An Extreme Case. Congenital Anomalies (Kyoto), 52, 59-61. http://dx.doi.org/10.1111/j.1741-4520.2010.00301.x

[7] Robin, N.H., Franklin, J., Prucka, S., Ryan, A.B. and Grant, J.H. (2005) Clefting, Amniotic Bands, and Polydactyly: A Distinct Phenotype That Supports an Intrinsic Mechanism for Amniotic Band Sequence. American Journal of Medical Genetics Part A, 137, 298-301. http://dx.doi.org/10.1002/ajmg.a.30885

[8] Sato, Y., Minagi, S., Akagawa, Y. and Nagasawa, T. (1989) An Evaluation of Chewing Function of Complete Denture Wearers. The Journal of Prosthetic Dentistry, 62, 50-53. http://dx.doi.org/10.1016/0022-3913(89)90047-4

[9] Matsui, Y., Ohno, K., Shirota, T., Imai, S., Yamashita, Y. and Michi, K. (1995) Speech Function Following Maxillectomy Reconstructed by Rectus Abdominis Myocutaneous Flap. Journal of Cranio-Maxillo-Facial Surgery, 23, 160164. http://dx.doi.org/10.1016/S1010-5182(05)80005-8 